\title{
Strong decay of scalar B meson
}

\author{
Benoît Blossier* \\ Laboratoire de Physique Théorique, CNRS, Univ. Paris-Sud, Université Paris-Saclay, F-91405 \\ Orsay Cedex, France \\ E-mail: benoit.blossiereth.u-psud.fr
}

The use of Heavy Meson Chiral Perturbation Theory is very popular to extrapolate to the chiral limit phenomenologically relevant quantities of heavy flavour physics that are computed on the lattice at non physical large pion masses. We show that the effect of the first orbital excitation may be not negligible in chiral loops because the corresponding pionic coupling is large. We have extracted that coupling by measuring single meson to multihadrons correlation function at lattice points where the scalar $B$ meson lies near the $B \pi$ threshold. That computation is required to obtain in the chiral limit the scalar $B$ meson decay constant in static limit of Heavy Quark Effective Theory.

The European Physical Society Conference on High Energy Physics 22-29 July 2015

Vienna, Austria

${ }^{*}$ Speaker. 


\section{Phenomenological introduction: ' $1 / 2$ vs. $3 / 2$ ' puzzle in $B \rightarrow D^{* *}$}

Properly treating the long-distance dynamics of QCD is of high relevance to control theoretical systematic effects on low-energy processes that are investigated at present and future colliders, in order to detect indirect effects of New Physics. It is particularly true for processes involving excited states, that occur often in experiments. An illuminating example of that assertion is the $b \rightarrow c$ transition and the answer to the long-standing question about the $\sim 3 \sigma$ discrepancy between $\left|V_{c b}\right|^{\text {excl }}$ and $\left|V_{c b}\right|^{\text {incl }}$ [1]: more specifically, studies have been led on the composition of the final hadronic state $X_{c}$ in the semileptonic decay $B \rightarrow X_{c} l v$. We collect in the left panel of Table 1 a couple of branching ratios: about $\sim 25 \%$ of the total width $\Gamma\left(B \rightarrow X_{c} l v\right)$ is neither $B \rightarrow D$ nor $B \rightarrow D^{*}$. $1 / 3$ of it comes from the channel $B \rightarrow D_{\text {narrow. Assuming a quite large } B \rightarrow D^{\prime} l v}$ width [2] and using the fact that $\Gamma\left(D^{\prime} \rightarrow D_{j_{l}=1 / 2} \pi\right) \gg \Gamma\left(D^{\prime} \rightarrow D_{j_{l}=3 / 2} \pi\right)$, one could observe an excess of $B \rightarrow\left(D_{1 / 2} \pi\right) l v$ events with respect to their $B \rightarrow\left(D_{3 / 2} \pi\right) l v$ counterparts. It has been asked whether a potentially large $B \rightarrow D^{\prime} l v$ width could also explain the "1/2 vs. 3/2" puzzle: $\left[\Gamma\left(B \rightarrow D_{1 / 2} l v\right) \simeq \Gamma\left(B \rightarrow D_{3 / 2} l v\right)\right]^{\exp }$ while $\left[\Gamma\left(B \rightarrow D_{1 / 2} l v\right) \ll \Gamma\left(B \rightarrow D_{3 / 2} l v\right)\right]^{\text {theory }}[3]$. A part of the suppression comes from a kinematical factor: indeed, $\frac{d \Gamma^{B \rightarrow D_{1 / 2}}}{d \Gamma^{B \rightarrow D_{3 / 2}}}=\frac{2}{(w+1)^{2}}\left(\frac{\tau_{1 / 2}(w)}{\tau_{3 / 2}(w)}\right)^{2}$. We have made in the center panel of Table 1 a comparison between theory and experiment. We see that the largest tension is for $B \rightarrow D_{0}^{*} l v$. The experimental side issues are the identification of the $D_{0}^{*}$ state and the disagreement in $\mathscr{B}\left(B \rightarrow D_{1}^{*} l v\right)$ between Belle (no events) and BaBar (claim of a signal). On the theory side, the limitation is that the predictions are made essentially in the infinite mass limit, including lattice QCD calculations of Isgur-Wise functions $\tau_{1 / 2}$ and $\tau_{3 / 2}$ [4], [5]. The non leptonic decays $B_{(s)} \rightarrow D_{(s)} \pi$ are presumably a more favorable situation. From the comparison between theory and experiment realised in the right panel of Table 1, we conclude that, despite a) an experimental disagreement in $\mathscr{B}\left(B_{d} \rightarrow D_{0}^{*} \pi\right)$ between Belle and BaBar, however not so conclusive, and b) the fact that theoretical predictions are based on the factorisation approximation (working well for the so called Class I decays), there is a much better agreement between theory and experiment for $B_{d} \rightarrow D_{0}^{*} \pi$ than for $B_{d} \rightarrow D_{0}^{*} l v$. More explicitly, the Class I process is expressed by

$\Gamma\left(\bar{B}^{0} \rightarrow D_{0}^{+*} \pi^{-}\right)=\frac{G_{F}^{2}}{8 \pi}\left|V_{c b} V_{u d}\right|^{2} \frac{1}{m_{B}^{2}} m_{D_{0}^{+*}}\left[\lambda\left(m_{B}, m_{D_{0}^{*}}, m_{\pi}\right)\right]^{1 / 2} \times\left[\left(m_{B}+m_{D_{0}^{*}}\right)\left(w_{\max }-1\right) a_{1} f_{\pi} \tau_{1 / 2}\left(w_{\max }\right)\right]^{2}$,

$$
\begin{aligned}
\mathscr{B}\left(B_{d} \rightarrow X_{c} l v\right)=(10.09 \pm 0.22) \% \\
\left.\mathscr{B}\left(B_{d} \rightarrow\left[\text { non }-D^{(*)}\right] l v\right)=2.86 \pm 0.25\right) \% \\
\mathscr{B}\left(B_{d} \rightarrow D_{\text {narrow }}^{* *} l v\right)=(0.87 \pm 0.06) \% \\
\mathscr{B}\left(B_{d} \rightarrow D^{(*)} \pi l v\right)=(1.43 \pm 0.08) \% \\
\mathscr{B}\left(B_{d} \rightarrow[D \pi]_{\text {broad }} l v\right)=(0.42 \pm 0.06) \% \\
\mathscr{B}\left(B_{d} \rightarrow\left[D^{*} \pi\right]_{\text {broad }} l v\right)=(0.33 \pm 0.07) \%
\end{aligned}
$$

\begin{tabular}{|c|c|}
\hline$B_{d} \rightarrow D^{* *} e v$ & $\mathscr{B}_{\exp } / \mathscr{B}_{\text {th }}$ \\
\hline$D_{2}^{*}$ & 0.5 \\
$D_{1}$ & 1 \\
$D_{1}^{*}$ & {$[0,5]$} \\
$D_{0}^{*}$ & $6 \pm 1$ \\
\hline
\end{tabular}

\begin{tabular}{|c|c|}
\hline$B_{d} \rightarrow D^{* *} \pi$ & $\mathscr{B}_{\exp } / \mathscr{B}_{\text {th }}$ \\
\hline$D_{2}^{*}$ & $\sim 0.5$ \\
$D_{1}$ & {$[0.5,1]$} \\
$D_{1}^{*}$ & no result \\
$D_{0}^{*}$ & {$[0.2,2.6]$} \\
\hline
\end{tabular}

Table 1: Branching ratio of $B \rightarrow X_{C} l v$ (left panel); comparison between theory and experiment for the different $B \rightarrow D^{* *} l v$ channels (center panel); comparison between theory and experiment for the different $B \rightarrow D^{* *} \pi$ channels (right panel). 


$$
\lambda(x, y, z)=\left[x^{2}-(y+z)^{2}\right]\left[x^{2}-(y-z)^{2}\right], \quad w=\frac{p_{B} \cdot p_{D_{0}^{*}}}{m_{B} m_{D_{0}^{*}}} .
$$

The factorised amplitude of the Class III decay normalised by the Class I decay reads

$$
\frac{A_{\text {fact }}^{I I I}}{A_{\text {fact }}^{I}}=\left[1+\frac{a_{2}}{a_{1}} \frac{m_{B}^{2}}{2 \sqrt{m_{B} m_{D_{0}^{*}}}\left(m_{B}+m_{D_{0}^{*}}\right)\left(w_{\max }-1\right)} \frac{f_{D_{0}^{*}}}{f_{\pi}} \frac{f^{B \rightarrow \pi}\left(m_{D_{0}^{*}}^{2}\right)}{\tau_{1 / 2}\left(w_{\max }\right)}\right] .
$$

The ratio of Wilson coefficients $a_{2} / a_{1}$ is extracted from $\frac{\mathscr{B}\left(B^{-} \rightarrow D^{0} \pi^{-}\right)}{\mathscr{B}\left(\bar{B}^{0} \rightarrow D^{+} \pi^{-}\right)}$, known experimentally [1], $f_{D_{0}^{*}} / f_{\pi}$ has to be extracted from lattice QCD simulations, $\tau_{1 / 2}\left(w_{\max }\right)$ might be extracted from $B_{s} \rightarrow D_{s}^{* *} \pi$. The $D_{s}$ sector is peculiar: indeed, $D_{s 0}^{*}(2317)$ and $D_{s 1}^{*}(2460)$ sit below the $D K$ and $D^{*} K$ thresholds: hence, they are narrow states. So, it is recommended to examine them because there is no experimental issue from their broadness. It has been proposed to study hadronic decays $B_{s} \rightarrow D_{s 0}^{*+}(2317) \pi^{-}$and $B_{s} \rightarrow D_{s 1}^{*+}(2460) \pi^{-}[6]:$ at LHCb, the number of expected events with 1 $\mathrm{fb}^{-1}$ of integrated luminosity is $N\left(B_{s} \rightarrow D_{s 0}^{*-} \pi^{+}\right) \sim 100$.

\section{Measurement of $f_{B_{0}^{*}}$}

Computing the $B_{0}^{*}$ decay constant in the static limit of Heavy Quark Effective Theory is a first step towards the extraction of $f_{D_{0}^{*}}$. We have used 2 kinds of interpolating fields $O_{B_{0}^{*}, \Gamma}^{n}(x)=$ $\left[\bar{\psi}_{l}^{(n)} \Gamma \psi_{h}\right](x)$ to couple to the scalar meson: a local one, corresponding to $\Gamma=\gamma_{0}$, and with a symmetrised derivative corresponding to $\Gamma=\sum_{i} \gamma_{i} \overleftarrow{\nabla}^{s}$. Moreover, $n$ levels of Gaussian smearing are applied on the light quark field. Our results are based on simulations with $O(a)$ improved $N_{f}=2$ Wilson-Clover fermions, whose the parameters are collected in Table 2: several lattice spacings and pion masses are considered to perform chiral and continuum extrapolations. We have extracted the $B_{0}^{*}$ mass and decay constant by solving a Generalized Eigenvalue Problem $C(t) v_{n}\left(t, t_{0}\right)=\lambda_{n}\left(t, t_{0}\right) C\left(t_{0}\right) v_{n}\left(t, t_{0}\right)$. The renormalised temporal vector $O(a)$ improved operator reads $\left(V_{R}\right)_{0}^{\text {stat }}(x)=Z_{V}^{\text {stat }}\left(1+b_{V}^{\text {stat }} a m_{q}\right)\left(V_{I}\right)_{0}^{\text {stat }}(x),\left(V_{I}\right)_{0}^{\text {stat }}(x)=V_{0}^{\text {stat }}(x)+c_{V}^{\text {stat }} \delta V(x)$, with $V_{0}^{\text {stat }}(x)=\bar{\psi}_{l}(x) \gamma_{0} \psi_{h}(x), \delta V(x)=\bar{\psi}_{l}(x) \gamma_{i} \overleftarrow{\nabla}_{i}^{s} \psi_{h}(x)$; the constants $Z_{V}^{\text {stat }}, c_{V}^{\text {stat }}$ and $b_{V}^{\text {stat }}$ are computed perturbatively. The bare matrix elements are given by the expressions

$$
f_{B_{0}^{*}}^{\text {stat }}\left(t, t_{0}\right)=R_{1}^{\mathrm{stat}}\left(t, t_{0}\right) \times\left(v_{1}^{\mathrm{stat}}\left(t, t_{0}\right), C_{V, \text { loc }}^{\mathrm{stat}}(t)\right), \quad f_{B_{0}^{*}}^{\mathrm{dV}}\left(t, t_{0}\right)=R_{1}^{\mathrm{stat}}\left(t, t_{0}\right) \times\left(v_{1}^{\mathrm{stat}}\left(t, t_{0}\right), C_{\delta V, \text { loc }}(t)\right) .
$$

The 2-pt correlation functions are given by

$$
\left(C_{V}\right)_{i j}=\left\langle\mathscr{O}_{i}(t) \mathscr{O}_{j}(0)\right\rangle, \quad\left(C_{V, \text { loc }}\right)_{i}=\left\langle V_{0}(t) \mathscr{O}_{i}(t)\right\rangle, \quad\left(C_{\delta V, \text { loc }}\right)_{i}=\left\langle\delta V(t) \mathscr{O}_{i}(0)\right\rangle
$$

\begin{tabular}{|c|c|c|c|c|}
\hline$\beta$ & $a[\mathrm{fm}]$ & $L / a$ & $m_{\pi}[\mathrm{MeV}]$ & $\Delta=m_{B_{0}^{*}}-\left(m_{B}+m_{\pi}\right)[\mathrm{MeV}]$ \\
\hline 5.2 & 0.075 & 48 & 280 & 100 \\
\hline 5.3 & 0.065 & 32 & 440 & -40 \\
& & 48 & 310 & 75 \\
\hline 5.5 & 0.048 & 48 & 440 & 65 \\
\hline
\end{tabular}

Table 2: Parameters of the lattice ensembles used in our analysis and difference between the mass of the scalar $B$ meson and the $B \pi$ state energy. 
Finally we have

$$
R_{n}^{\mathrm{stat}}=\left(v_{n}^{\mathrm{stat}}\left(t, t_{0}\right), C_{V}(t) v_{n}^{\mathrm{stat}}\left(t, t_{0}\right)\right)^{-1 / 2}\left(\frac{\lambda_{n}\left(t, t_{0}\right)}{\lambda_{n}\left(t+1, t_{0}\right)}\right)^{t /(2 a)}
$$

We have obtained $\Delta m_{B_{0}^{*}} \equiv m_{B_{0}^{*}}-m_{B}$ at the physical point by using the following extrapolation formula: $\Delta m_{B_{0}^{*}}\left(m_{\pi}, a\right)=A+B\left(y-y^{\text {phys }}\right)+D_{\mathrm{HYP} i}\left(a / a_{\beta=5.3}\right)^{2}$, with $y=\frac{m_{\pi}^{2}}{8 \pi^{2} f_{\pi}^{2}}$; HYP1 and HYP2 are 2 different HQET discretisations. A constant fit in $m_{\pi}^{2}$ gives $\Delta m_{B_{0}^{*}}=371$ (33) MeV while, with a linear fit, we get $\Delta m_{B_{0}^{*}}=399(17) \mathrm{MeV}$. They are sketched in Figure 1. Taking the average value of both fits and including the discrepancy as a systematic error, our preliminary value is $\Delta m_{B_{0}^{*}}=385(33)(14) \mathrm{MeV}$. As we work with 2 dynamical flavours, we have to worry about the possible mixing of $B_{0}^{*}$ state with $B \pi$ multihadronic state in $S$ wave. Indeed, our simulation points lie close the threshold: we tabulate the value of $\Delta=m_{B_{0}^{*}}-\left(m_{B}+m_{\pi}\right)$ in the last column of Table 2. We have computed the matrix of correlators $C(t)=\left[\begin{array}{cc}C_{B_{0}^{*} B_{0}^{*}}(t) & C_{B_{0}^{*} B \pi}(t) \\ C_{B \pi} B_{0}^{*}(t) & C_{B \pi} B \pi\end{array}\right]$ for the lattice ensemble where the $B \pi$ threshold is the closest from $B_{0}^{*}$. Diagrams of the different correlators are depicted in Figure 2. Even if the contribution of box and cross diagrams is small compared to the direct diagram in $C_{B \pi B \pi}, 0.1 \%$ and $1 \%$ respectively, their impact is not totally absent. Moreover, we show in Figure 3 that $B_{0}^{*}$ and $B \pi$ states are less degenerate when the off-diagonal terms are introduced in the matrix of correlators. However the effect is small enough to neglect it at a level of precision $\sim$ $10 \%$ on $f_{B_{0}^{*}}$ we hope. It is expressed by $a^{3 / 2} f_{B_{0}^{*}} \sqrt{\frac{m_{B_{0}^{*}}}{2}}=Z_{V}^{\text {stat }}\left(1+b_{V}^{\text {stat }} a m_{q}\right)\left[f_{B_{0}^{*}}^{\text {stat }}+c_{V}^{\text {stat }} f_{B_{0}^{*}}^{\delta V}\right]$ and is extrapolated to the physical point with the following fit formula:

$f_{B_{0}^{*}}\left(m_{\pi}, a, \mathrm{HYP} i\right)=\alpha+\beta\left(y-y^{\text {phys }}\right)+\gamma_{\mathrm{HYP} i}\left(a / a_{\beta=5.3}\right)^{2}$. The quality of the fit is shown in the right

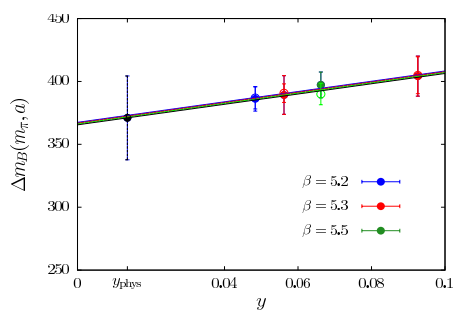

(a)

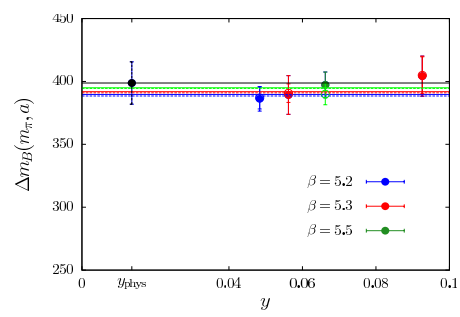

(b)

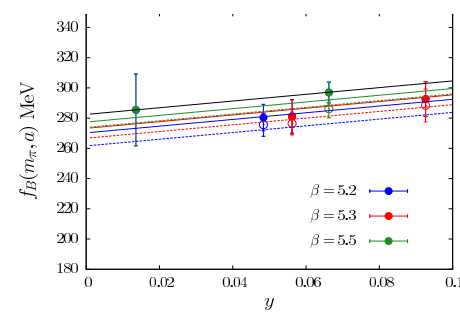

(c)

Figure 1: Extrapolation to the physical point of $m_{B_{0}^{*}}-m_{B}$ by a constant fit in $m_{\pi}^{2}$ (a) and a linear fit in $m_{\pi}^{2}$ (b); extrapolation to the physical point of $f_{B_{0}^{*}}$ (c).

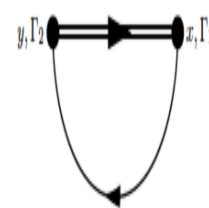

(a)

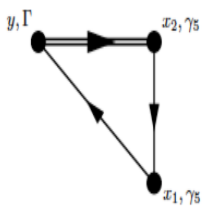

(b)

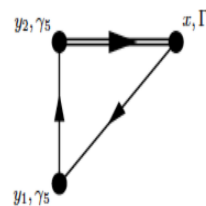

(c)
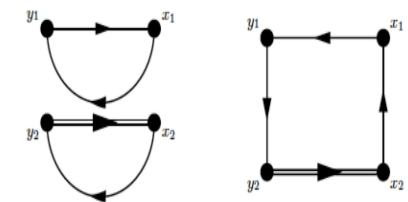

(d)

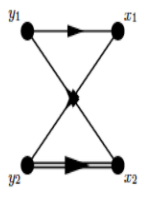

Figure 2: Diagrams of the correlator $C_{B_{0}^{*} B_{0}^{*}}$ (a), $C_{B_{0}^{*} B \pi}$ (b), $C_{B \pi B_{0}^{*}}$ (c) and $C_{B \pi B \pi}$ (d). 
panel of Figure 1. Our preliminary value is $f_{B_{0}^{*}}=285(23) \mathrm{MeV}$, in agreement with a first lattice estimate $f_{B_{0}^{*}}=294(88) \mathrm{MeV}[7]$.

\section{Pion couplings to $B_{0}^{*}$}

Heavy Meson Chiral Perturbation Theory is often used to extrapolate lattice data in the heavylight sector. The HM $\chi$ PT Lagrangian reads

$$
\begin{aligned}
\mathscr{L}_{\mathrm{HM} \chi \mathrm{PT}} & =\frac{f_{\pi}^{2}}{8} \operatorname{Tr}\left(\partial^{\mu} \Sigma \partial_{\mu} \Sigma^{\dagger}\right)+i \operatorname{Tr}(H v \cdot \mathscr{D} \bar{H})+i \operatorname{Tr}(S v \cdot \mathscr{D} \bar{S}) \\
& +i \hat{g} \operatorname{Tr}\left(H \gamma_{\mu} \gamma_{5} \mathscr{A}^{\mu} \bar{H}\right)+i \tilde{g} \operatorname{Tr}\left(S \gamma_{\mu} \gamma_{5} \mathscr{A}^{\mu} \bar{S}\right)+i h \operatorname{Tr}\left(S \gamma_{\mu} \gamma_{5} \mathscr{A}^{\mu} \bar{H}\right),
\end{aligned}
$$

where $\hat{g}, \tilde{g}$ and $h$ are low energy constants. The decay $B_{0}^{* 0} \rightarrow B^{+} \pi^{-}$is expressed by

$$
\Gamma\left(B_{0}^{* 0} \rightarrow B^{+} \pi^{-}\right)=\frac{1}{8 \pi} g_{B_{0}^{*} B \pi}^{2} \frac{\left|\vec{q}_{\pi}\right|}{m_{B_{0}^{*}}^{2}}, \quad\left|\vec{q}_{\pi}\right|=\frac{\sqrt{\left[m_{B_{0}^{*}}^{2}-\left(m_{B}+m_{\pi}\right)^{2}\right]\left[m_{B_{0}^{*}}^{2}-\left(m_{B}-m_{\pi}\right)^{2}\right]}}{2 m_{B_{0}^{*}}} .
$$

In terms of pionic couplings, it reads also

$$
\Gamma\left(B_{0}^{*} \rightarrow B^{+} \pi^{-}\right)=\frac{h^{2}}{8 \pi f_{\pi}^{2}} \frac{m_{B}}{m_{B_{0}^{*}}^{3}}\left(m_{B_{0}^{*}}^{2}-m_{B}^{2}\right)^{2}\left|\vec{q}_{\pi}\right|, \quad g_{B_{0}^{*} B \pi}=\sqrt{\frac{m_{B}}{m_{B_{0}^{*}}}}\left(m_{B_{0}^{*}}^{2}-m_{B}^{2}\right) \frac{h}{f_{\pi}} .
$$

Instead of extracting $h$ from the density distribution [8], we have considered the ratio $C_{B_{0}^{*} B \pi}^{(2)}(t) / \sqrt{C_{B_{0}^{*} B_{0}^{*}}^{(2)}(t) C_{B \pi B \pi}^{(2)}(t)}[9]$. A byproduct of our analysis is the extraction of $\tilde{g}$ using standard GEVP and sGEVP methods as described in [10], [11]. Extrapolations at the physical point performed with a constant fit, a linear fit in $m_{\pi}^{2}$ and NLO formulae of HM $\chi$ PT [12], [8], lead to the results $h=0.84(3)(2)$ and $\tilde{g}=0.122(8)(6)$. We have collected in Figure 4 different estimates of $h$ : experimental width $\Gamma\left(D_{0}^{*}\right)$ [1], lattice measurement through the phase shift in $D \pi$ scattering state [13] (both, under the hypothesis of small $1 / m_{c}$ corrections), QCD sum rules [14], [15], density distribution of the axial current [8], transition at the threshold $m_{B_{0}^{*}} \sim E_{B \pi}$ [9]. A word about the Adler-Weisberger sum rule:

$$
\sum_{\delta}\left|X_{B \delta}\right|^{2}=1, \Gamma(\mathscr{I} \rightarrow \mathscr{F} \pi)=\frac{1}{2 \pi f_{\pi}^{2}} \frac{|\vec{q}|^{3}}{2 j_{\mathscr{I}}+1}\left|X_{\mathscr{I}} \rightarrow \mathscr{F}\right|^{2} .
$$

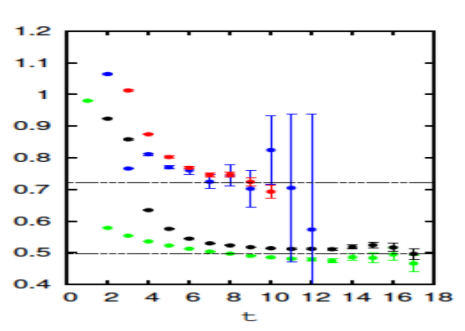

(a)

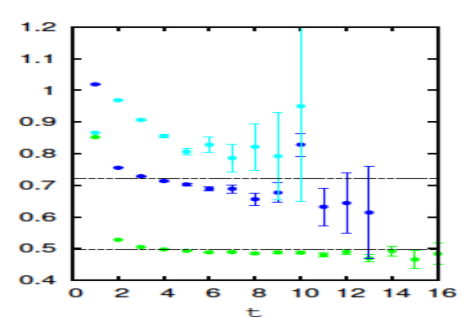

(b)

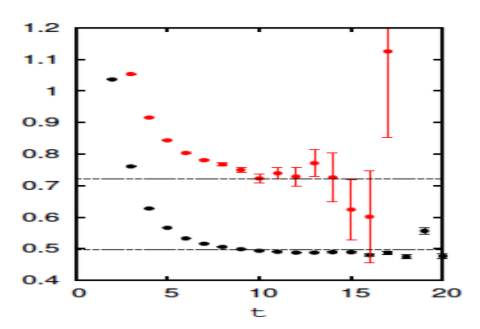

(c)

Figure 3: Effective masses extracted on the full basis of interpolating fields $\left\{B_{0}^{*} B \pi\right\}$ (a), $B_{0}^{*}$ (b) and $B \pi$ (c). 
With $\hat{g} \sim 0.5$, it is almost saturated by $B^{*}$ and $B_{0}^{*}$. $\tilde{g}$ is much smaller than the other pion couplings; $h$ is pretty large: it means that some care is required in the application of $\mathrm{HM} \chi \mathrm{PT}$ for pion masses close to $m_{B_{0}^{*}}-m_{B} \sim 400 \mathrm{MeV}: B$ meson orbital excitations degrees of freedom can not be neglected in chiral loops.

\section{References}

[1] K.A. Olive et al. [Particle Data Group], Chin. Phys. C 38, 090001 (2014).

[2] F. U. Bernlochner, Z. Ligeti and S. Turczyk, Phys. Rev. D 85 (2012) 094033.

[3] A. Le Yaouanc et al, Phys. Rev. D 56 (1997) 5668; A. K. Leibovich et al, Phys. Rev. D 57 (1998) 308; D. Becirevic et al, Phys. Rev. D 87, no. 5, 054007 (2013); I. I. Bigi et al, Eur. Phys. J. C 52, 975 (2007).

[4] D. Becirevic, B. Blossier, P. Boucaud, G. Herdoiza, J. P. Leroy, A. Le Yaouanc, V. Morenas and O. Pene, Phys. Lett. B 609, 298 (2005).

[5] B. Blossier et al. [European Twisted Mass Collaboration], JHEP 0906, 022 (2009).

[6] D. Becirevic, A. Le Yaouanc, L. Oliver, J. C. Raynal, P. Roudeau and J. Serrano, Phys. Rev. D 87, no. 5, 054007 (2013).

[7] G. Herdoiza et al. [UKQCD Collaboration], Phys. Rev. D 74, 014510 (2006).

[8] D. Becirevic, E. Chang and A. Le Yaouanc, arXiv:1203.0167 [hep-lat].

[9] B. Blossier, N. Garron and A. Gérardin, Eur. Phys. J. C 75, no. 3, 103 (2015).

[10] J. Bulava, M. Donnellan and R. Sommer, JHEP 1201, 140 (2012).

[11] B. Blossier, J. Bulava, M. Donnellan and A. Gérardin, Phys. Rev. D 87, no. 9, 094518 (2013).

[12] W. Detmold, C.-J. D. Lin and S. Meinel, Phys. Rev. D 84, 094502 (2011).

[13] D. Mohler, S. Prelovsek and R. M. Woloshyn, Phys. Rev. D 87, no. 3, 034501 (2013).

[14] P. Colangelo, F. De Fazio, G. Nardulli, N. Di Bartolomeo and R. Gatto, Phys. Rev. D 52, 6422 (1995).

[15] T. M. Aliev and M. Savci, J. Phys. G 22, 1759 (1996), [J. Phys. G 22, 1759 (1996)].

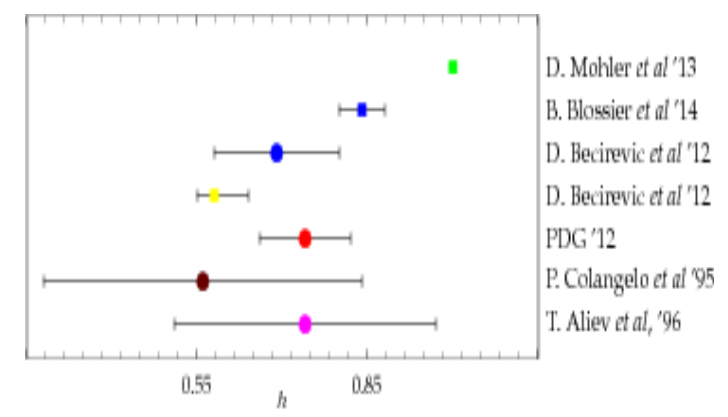

Figure 4: Collection of results for the coupling $h$. 